\title{
Beyond the fluid bolus
}

\author{
Peter Bentzer ${ }^{1,2}$, Anja Lindén-Søndersø $\varnothing^{1,2}$ \\ ${ }^{1}$ Department of Anesthesia and Intensive Care, Helsingborg Hospital, Helsingborg, Sweden; ${ }^{2}$ Department of Clinical Sciences Lund, Anesthesiology \\ and Intensive Care, Lund University, Lund, Sweden \\ Correspondence to: Peter Bentzer, Department of Anesthesiology and Intensive Care, Helsingborg Hospital, Charlotte Yhlens gata 10,25187 \\ Helsingborg, Sweden. Email: peter.bentzer@med.lu.se. \\ Provenance: This is an invited article commissioned by the Section Editor, Xue-Zhong Xing, MD [National Cancer Center (NCC)/Cancer Hospital, \\ Chinese Academy of Medical Sciences (CAMS) and Peking Union Medical College (PUMC), Beijing, China]. \\ Comment on: Roger C, Zieleskiewicz L, Demattei C, et al. Time course of fluid responsiveness in sepsis: the fluid challenge revisiting (FCREV) study. \\ Crit Care 2019;23:179.
}

Submitted Nov 27, 2019. Accepted for publication Dec 04, 2019.

doi: $10.21037 /$ jtd.2019.12.34

View this article at: http://dx.doi.org/10.21037/jtd.2019.12.34

In a recent study published in Critical Care, Roger and colleagues (1) evaluated the time course of the response to a fluid bolus in patients suffering from septic shock in 11 intensive care units in France. The authors prospectively included adult patients with septic shock in whom the physician caring for the patient planned a fluid bolus due to persistent hypotension, decreased central venous oxygenation, decreased urine output, skin mottling or increased lactate. Following transthoracic Doppler echocardiography (TTE) the patients received a $500 \mathrm{~mL}$ bolus of $0.9 \% \mathrm{NaCl}$ in $10 \mathrm{~min}$. A TTE was then performed at 10, 20 and $30 \mathrm{~min}$ after start of the fluid bolus $\left(\mathrm{T}_{10}, \mathrm{~T}_{20}\right.$ and $\left.\mathrm{T}_{30}\right)$. The change in stroke volume was assessed by measurement of the sub aortic velocity time integral (VTI) at the different time points and an increase in VTI by $>15 \%$ was considered to represent an equal increase in stroke volume: i.e., the patient was a fluid responder. At $\mathrm{T}_{10} 53 \%$ of the patients were responders. Of these patients only $51 \%$ were classified as responders at $T_{30}$ and the authors called these patients persistent responders. Of the patients that were classified as non-responders at $T_{10} 6 \%$ were classified as responders at $\mathrm{T}_{30}$. This means that only $30 \%$ of the included patients were responders when assessed at $T_{30}$. The study was well designed and the indications for fluid administration in the study are similar to those used in daily practice (2). The data adds to previous data showing that the effect of a crystalloid fluid bolus on cardiac output in septic shock is short lived and returns to baseline within $1 \mathrm{~h}$ hour after completion of the bolus in patient whom were initially responders (3).

Given the increased awareness in the critical care community of side-effects of liberal fluid resuscitation in septic shock we believe these results raise some intriguing questions which warrants a commentary. How do we explain the transient nature of the response in responders? The objective of a fluid bolus is to increase cardiac output and a fluid bolus is presumed to achieve this objective by increasing venous return through an increase in plasma volume. It follows that the duration of the plasma volume expansion obtained by the fluid bolus could have an impact on the duration of the response. From this perspective it is of interest to note that maximum plasma volume expansion following a rapid infusion of a crystalloid can be seen immediately after completion of the bolus. In the ensuing 25-30 min a rapid redistribution occurs (4). It is increasingly recognized that the duration and magnitude of the plasma volume expansion after the redistribution phase following a fluid bolus is context dependent. This means that in a hypovolemic patient with intact homeostatic mechanisms, such as following a hemorrhage or dehydration, compensatory mechanism will add to the plasma volume expansion obtained by the fluid bolus resulting in a large and sustained increase in plasma volume (5-7). During these conditions, plasma volume may increase by $20-25 \%$ of the infused volume after the initial distribution. This is, in contrast to septic shock, a condition in which disrupted homeostatic mechanisms and an increase in the extravascular distribution volume most likely contributes 
to the pathophysiology. During these conditions plasma volume expansion after the initial distribution may be in the range of $1-6 \%(5,8)$. Based on the above we believe the that a transient response in some patients is an expected finding.

Is it possible that the choice of resuscitation fluid could have influenced the number of responders at $T_{30}$ ? It is well established that colloids are more efficacious as plasma volume expanders than crystalloids and in sepsis the immediate plasma volume expansion of $5 \%$ albumin is about 5 times higher that of a $0.9 \% \mathrm{NaCl}$ (9). To our knowledge there are no clinical studies available comparing the duration of the plasma volume expansion by albumin to that of crystalloids in sepsis. However, data from a small study on patients with septic shock suggests that plasma volume expansion following resuscitation with $20 \%$ albumin persists unchanged for at least $2 \mathrm{~h}$ after completion of resuscitation (10). Similarly, in a recent study on postoperative patients after major abdominal surgery plasma volume expansion persisted unchanged for at least $2.5 \mathrm{~h}$ after a bolus infusion of $5 \%$ albumin at a dose of $10 \mathrm{~mL} / \mathrm{kg}$ (11). These data collectively suggest that choice of fluid may have influenced the result and highlight the need for studies comparing fluid response over time after resuscitation with albumin or crystalloids.

The increasing awareness of potential adverse effects of fluid administration has led to a surge of studies investigating methods to predict fluid responsiveness. In the majority of these studies the inclusion criteria has been very similar to those employed in the study by Roger and colleagues. As mentioned above, the fraction of responders immediately after completion of the fluid bolus dose was about $50 \%$ in the study by Roger. This is a remarkably consistent figure across a large number of studies investigating various predictors of fluid responsiveness in critically ill patients after the initial resuscitation $(12,13)$. Of the 50 studies included in a meta-analysis by Bentzer et al. (12) evaluating different predictors of fluid responsiveness, the majority either did not describe in detail when the response was assessed or stated that it was performed immediately after the bolus. In 3 studies, the response was evaluated 30 min after completion of the bolus. The results by Roger and colleagues indicate that many previous studies investigating predictors of fluid responsiveness may have included both transient and persistent responders in the group of patients deemed to be responders. Given that one of the purposes of assessing fluid responsiveness it to limit unnecessary fluid administration the question could be asked if a short-lasting increase in cardiac output after a fluid bolus is worth the price of the risks associated with a positive fluid balance (14)? The results provided by Roger and colleagues suggest that future studies evaluating methods to assess fluid responsiveness should consider evaluating the response to fluids at later time points, perhaps even beyond $30 \mathrm{~min}$, to identify the persistent responders. These patients are likely to have a more favorable risk-benefit ratio with regards to fluids. Unfortunately, no attempt was made to predict persistent responders in the study by Roger and colleagues and future studies will have to assess this issue. Should such predictors become available it is possible that the potential to limit unnecessary fluid administration is even higher than previously thought.

\section{Acknowledgments}

None.

\section{Footnote}

Conflicts of Interest: The authors have no conflicts of interest to declare.

Ethical Statement: The authors are accountable for all aspects of the work in ensuring that questions related to the accuracy or integrity of any part of the work are appropriately investigated and resolved.

\section{References}

1. Roger C, Zieleskiewicz L, Demattei C, et al. Time course of fluid responsiveness in sepsis: the fluid challenge revisiting (FCREV) study. Crit Care 2019;23:179.

2. Cecconi $\mathrm{M}$, Hofer $\mathrm{C}$, Teboul JL, et al. Fluid challenges in intensive care: the FENICE study: A global inception cohort study. Intensive Care Med 2015;41:1529-37.

3. Nunes T, Ladeira R, Bafi A, et al. Duration of hemodynamic effects of crystalloids in patients with circulatory shock after initial resuscitation. Ann Intensive Care 2014;4:25.

4. Hahn R. Volume kinetics for infusion fluids. Anesthesiology 2010;113:470-81.

5. Bansch P, Statkevicius S, Bentzer P. Plasma volume expansion with $5 \%$ albumin compared to Ringer's acetate during normal and increased microvascular permeability in the rat. Anesthesiology 2014;121:817-24.

6. Drobin D, Hahn RG. Volume kinetics of Ringer's solution in hypovolemic volunteers. Anesthesiology 1999;90:81-91. 
7. Statkevicius S, Frigyesi A, Bentzer P. Effect of ringers acetate in different doses on plasma volume in rat models of hypovolemia. Intensive Care Med Exp 2017;5:50.

8. Bark B, Öberg C, Grände PO. Plasma volume expansion by $0.9 \% \mathrm{NaCl}$ during sepsis/systemic inflammatory response syndrome, after hemorrhage, and during a normal state. Shock 2013;40:59-64.

9. Ernest D, Belzberg AS, Dodek PM. Distribution of normal saline and 5\% albumin infusions in septic patients. Crit Care Med 1999;27:46-50.

10. Margarson M, Soni N. Plasma volume measurement in septic patients using an albumin dilution technique: comparison with the standard radio-labelled albumin method. Intensive Care Med 2005;31:289-95

11. Statkevicius S, Bonnevier J, Fisher J, et al. Albumin

Cite this article as: Bentzer P, Lindén-Søndersø A. Beyond the fluid bolus. J Thorac Dis 2019;11(12):5685-5687. doi: 10.21037/ jtd.2019.12.34 infusion rate and plasma volume expansion: a randomized clinical trial in postoperative patients after major surgery. Crit Care 2019;23:191.

12. Bentzer P, Griesdale DE, Boyd J, et al. Will This Hemodynamically Unstable Patient Respond to a Bolus of Intravenous Fluids? JAMA 2016; 316:1298-309.

13. Messina A, Dell'Anna A, Baggiani M, et al. Functional hemodynamic tests: a systematic review and a metanalysis on the reliability of the end-expiratory occlusion test and of the mini-fluid challenge in predicting fluid responsiveness. Crit Care 2019;23:264.

14. Boyd JH, Forbes J, Nakada TA, et al. Fluid resuscitation in septic shock: a positive fluid balance and elevated central venous pressure are associated with increased mortality. Crit Care Med 2011;39:259-65. 